\title{
On the Instability and Folding Deformation of a Layered Viscoelastic Medium in Compression
} Consultant, Shell Development
Company, New York, N. Y.

Mem. ASME

\begin{abstract}
When a layer of material embedded in an infinite medium is subject to a compression parallel with the layer an instability tends to develop which manifests itself in the folding of the layer. This phenomenon is examined here for the general case where the layer and the surrounding medium are both viscoelastic. This problem which was examined in preliminary form in an earlier publication $[6]^{1}$ is treated here with particular attention to the effect of interfacial adherence of the layer and the medium, and to an evaluation of the amplitude of the folding. In general there is a lower and upper-critical value of the compressive load between which folding occurs with a finite rate of deformation. There appears also a dominant wave length, for which the rate of folding is maximum under a given load. The dominunt wave length may or may not depend on the load. The effect of interfacial adherence while not negligible is not generally significant. The rate of folding increases very rapidly beyond a certain value of the viscosity ratio of the two media. A brief discussion is also included of the thermodynamic implications of incremental stress-strain relations in prestressed media.
\end{abstract}

C onsider an inhomogeneous elastic medium of infinite extent containing a layer of material of higher rigidity. It is well known that such a system when subject to a compression parallel with the layer will buckle beyond a certain critical value of the load. This buckling instability manifests itself through a folding of the layer which appears suddenly with a characteristic wave length at the critical load. ${ }^{2}$ The nature of the phenomenon is typified in the elementary treatment of the buckling of a beam or an elastic foundation. It is clear that this phenomenon is a particular case of the much more general one where the layer and the medium are viscoelastic. In this case we shall not in general observe a sharp buckling, but a folding of the layer will appear with a rate of folding and a wave-length content which are dependent on the physical properties, the compressive load, and the initial irregularities of the layer.

The present paper is one in a series presenting the results of an investigation of these phenomena carried out at the Shell Development Company during the past 10 years. The subject is of course of primary interest to the geologist since it furnishes for the first time a quantitative basis for the evaluation of folding and other tectonic features of stratified rock formations. There are also interesting technological applications connected with the buckling failure of inhomogeneous structural material such as sandwich panels under conditions of creep and the deformation due to instability of materials in general in the viscoelastic temperature range when there is a high-temperature gradient. The

${ }^{1}$ Numbers in brackets designate References at end of paper.

2 The buckling of layered elastic media was investigated by Bijlaard $[1,2]$, Gough, Elam, and DeBruyne [3], Goodier [12], and others.

Presented at the Summer Conference of the Applied Mechanics Division, Troy, N. Y., June 18-20, 1959, of The AMERICAN SocIETY of Mechanical Engineers.

Discussion of this paper should be addressed to the Secretary, ASME, 29 West 39th Street, New York, N. Y., and will be accepted until October 10, 1959, for publication at a later date. Discussion received after the closing date will be returned.

Note: Statements and opinions advanced in papers are to be understood as individual expressions of their authors and not those of the Society. Manuscript received by ASME Applied Mechanics Division, March 11, 1958. Paper No. 59-APM-21. result also throws light on the behavior of such engineering components as an axially compressed rod in a viscoelastic fluid, and so on.

A discussion of the fundamental aspects of the theory is given in [6]. This makes use of the general results established previously for the viscoelastic behavior of solids on the basis of irreversible thermodynamics $[4,5]$. In the present paper we have emphasized a more exact approach which takes into account the adherence between the layer and the surrounding medium. While this adherence does not affect the phenomenon qualitatively, it does introduce considerably more complication in the analysis. We also have given a more complete discussion of some aspects of the phenomenon, and in particular of the magnitude of the instability as represented by the rate of growth of the folding. The theory is developed here by representing the layer as a viscoelastic plate obeying the equations derived from a straightforward generalization of the classical equations for elastic plates by a correspondence rule [5]. A more rigorous treatment of the problem on the basis of a continuum also has been completed [7]. This more rigorous treatment takes into account the existence of a compression in the surrounding medium which is neglected in the present theory. Results indicate that use of the plate equations is satisfactory for most practical purposes. In more recent work the effect of gravity has also been introduced [13].

In Section 1 we derive the equations of flexure of a viscoelastic plate under compression, and Section 2 derives the properties of the surrounding medium. These results are combined in Section 3 and equations are established for an embedded layer with perfect or imperfect adherence at the interface. We assume that if an interfacial slip occurs the friction is proportional to the rate of slip. Section 4 develops quantitatively the results for the case where the layer and the surrounding medium are both incompressible viscous solids under the assumption of perfect adherence. Particular attention is paid to a comparison with the case of perfect slip and to the rate of growth of the folding.

Section 5 discusses briefly other cases such as the elastic layer in a viscous medium, and two Maxwell materials. These other cases were treated in more detail in reference [6]. 
In Section 6 a few remarks are presented regarding the incremental stress-strain relations in nonlinear rheology and it is pointed out that an essential distinction is to be made between thermodynamic systems in the vicinity of equilibrium or in a steady state of flow.

It is pointed out that there exists, in general, a lower and higher limit for the critical compressive load. Between these limits the folding takes place with a dominant wave length; i.e., one for which under a given load there is a maximum rate of growth. The dominant wave length may or may not depend on the load. The latter case is true for two viscous fluids and in general for two materials with the same relaxation time. The rate of growth of the folds which is evaluated for the particular case of two viscous fluids becomes large and very wave-length-selective for values of the viscosity ratio $\mu / \mu_{1}$, of the order of about 70 or higher. The influence of interfacial adherence is found to be very small in the range where the magnitude of the instability becomes significant.

\section{Equations for Deformation of a Viscoelastic Plate}

We shall first derive the approximate equations for an elastic plate under the assumption that the wave length of the deformation is large relative to the thickness $h$ of the plate. We consider a two-dimensional deformation such that the upward displacement of the plate is, Fig. 1,

$$
v=v^{\prime} \cos l x
$$

The assumption stated above corresponds to the conditions $l h \ll 1$.

The upward plate deflection may be derived in terms of the bending moment $M$ per unit width. We may write the differential equation

$$
\frac{B h^{3}}{12} \frac{d^{2} v}{d x^{2}}=-M
$$

The sign of $M$ is taken positive clockwise when matter is located to the left of the cross section. The coefficient $B$ is

$$
B=\frac{4 G(G+\lambda)}{2 G+\lambda}
$$

The Lamé constants of the plate are denoted by $\lambda$ and $G .{ }^{3}$

The bending moment $M$ will now be determined. We assume that the plate is subject to a total compressive load $P h$ acting in its plane. In addition we assume a vertical load $q$ per unit area taken positive upward and a tangential force $\tau$ per unit area on the surface of the plate acting positively to the right on the upper surface and to the left on the lower surface, Fig. 1. These loads are distributed sinusoidally according to

$$
\begin{aligned}
& q=q_{0} \cos l x \\
& \tau=\tau_{0} \sin l x
\end{aligned}
$$

If we call $M$, the bending moment due to $q$, we may write

$$
\frac{d^{2} M_{1}}{d x^{2}}=-q
$$

${ }^{3}$ The shear modulus $G$ is also generally represented by $\mu$ but we prefer to reserve the latter symbol for the viscosity coefficient.

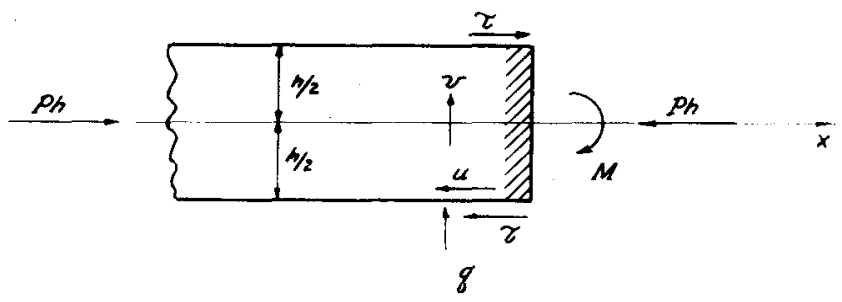

Fig. 1 Forces and displacements for the plate
The moment $M_{2}$ due to the tangential load must satisfy

$$
h \int_{0}^{x} \tau d x+M_{2}=M_{0}
$$

where $M_{0}$ is the moment at a cross section $x=0$ where $\tau=0$; i.e., the total shear also vanishes at that cross section. Differentiating (6) we find

$$
\frac{d M_{2}}{d x}=-\tau h
$$

Finally, we must evaluate the bending moment due to the axial compressive load $P h$. This bending moment depends on the deformation and is equal to

$$
M_{3}=P h v
$$

The total bending moment is

$$
M=M_{1}+M_{2}+M_{3}
$$

Combining (5), (7), (8), and (9) we derive

$$
\frac{d^{2} M}{d x^{2}}=-q-h \frac{d \tau}{d x}+P h \frac{d v^{2}}{d x^{2}}
$$

and substituting in (2) we find the differential equation for the plate deflection as

$$
\frac{1}{12} B h^{3} \frac{d^{4} v}{d x^{4}}+P h \frac{d^{2} v}{d x^{2}}=q+h \frac{d \tau}{d x}
$$

In addition to the plate deflection we also need for our present purpose the displacement $u$ of the plate tangentially to the surface. The sign of $u$ is chosen positive to the right on the upper surface. The displacement $u$ is due to the rotation $(d v) /(d x)$ of the cross section and in addition to the shearing deformation of the plate produced by the tangential force $\tau$. The sum of these two effects is

$$
u=-\frac{h}{2} \frac{d v}{d x}+\frac{h}{2} \frac{\tau}{G}
$$

It should be noted that in establishing the last relation we have assumed that the shearing deformation of the plate is solely due to $\tau$. There is, of course, an additional transverse shear associated with the bending which we have neglected. Although these two assumptions are not quite consistent, we are concerned here mainly with the separate evaluation of the effect of interfacial adherence and therefore we wish to introduce this effect as the sole correction to the approximate theory of reference [6] for the purpose of comparison. A consistent assumption would be to neglect the shear deformation of the plate. This is easily done by putting $G=\infty$ in relation (12) and $Q=\infty$ in all relations derived in the following. Further remarks on this point will be found in Section 4 . It should be added that the effect of shearing deformation associated with bending is evaluated implicitly in the exact treatment of reference [7] based on equations for the prestressed continuum. The magnitude of the effect is found to he not very significant.

Putting

we find

$$
\begin{gathered}
u=U \sin l x \\
v=V \cos l x \\
q_{0}=\left(\frac{1}{12} B h^{2} l^{2}-P+G\right) h l^{2} V-2 G l U \\
2 \tau_{0}=-2 G l V+\frac{4 G}{h} U
\end{gathered}
$$


As we have pointed out in earlier publications $[4,5,6]$, equations obtained for an elastic body can be extended readily to a viscoelastic continuum by replacing the elastic constants by their corresponding operators. We have called this the "correspondence rule." This rule holds for the most general case of anisotropy and is valid for relations derived by energy or variational methods. For an isotropic viscoelastic material Hooke's law is replaced by

$$
\sigma_{i j}=2 \bar{Q} e_{i j}+\delta_{i j} \bar{R} e
$$

Where $\sigma_{i j}$ and $e_{i j}$ are the stress and strain tensors, $\delta_{i j}$ is the Kronecker symbol and

$$
e=e_{x x}+e_{y y}+e_{x z}
$$

is the dilatation. The symbols $\bar{Q}$ and $\tilde{R}$ represent operators. They replace the Lamé constants $G$ and $\lambda$. We have shown [4] from linear irreversible thermodynamics that these operators are of the form

$$
\begin{aligned}
\bar{Q} & =\int_{0}^{\infty} \frac{p}{p+r} Q(r) \gamma(r) d r+Q+p Q^{\prime} \\
\bar{R} & =\int_{0}^{\infty} \frac{p}{p+r} R(r) \gamma(r) d r+R+p \bar{R}^{\prime}
\end{aligned}
$$

In these expressions $p$ is a differential time operator

$$
p=\frac{d}{d t}
$$

The variable of integration $r$ is a relaxation constant with a spectral density $\gamma(r)$. We have written the function $Q(r) \gamma(r)$ and $R(r) \gamma(r)$ with the spectral density function $\gamma(r)$ as a common factor in order to bring out the common properties of these functions as regards their singularities. In case of relaxation with a discrete spectrum the integrals are replaced by summations,

$$
\begin{aligned}
& \bar{Q}=\sum^{s} \frac{p}{p+r_{s}} Q_{s}+Q+p Q^{\prime} \\
& \bar{R}=\sum^{s} \frac{p}{p+r_{s}} R_{s}+R+p R^{\prime}
\end{aligned}
$$

It was also shown that the constants in the operator $\vec{Q}$ are all positive. In addition, we must have $\tilde{R}+\bar{Q}>0$. The applicability of these operators for incremental stresses in a linear or nonlinear prestressed medium is discussed in Section 6.

The significance of the operational stress-strain law (15) is multifold. It may be interpreted as a relation between Laplace or Fourier transforms of the stress and strain components. For instance, if the strain components are harmonic functions of time and represented by $e_{i j} e^{i \omega t}$ then the stress tensor is $\sigma_{i j} e^{i \omega t}$ where $e_{i j}$ is related to $\sigma_{i j}$ by equations (15) after putting $p=i \omega$ in the expression of the operators.

Relations (15) may also be considered as giving directly the response to an arbitrary function of time by operational rules or Fourier integrals. For instance, assume a relation for pure shear

$$
\sigma_{s}=2 \bar{Q} e_{s}
$$

and assume the shear strain to be of constant unit value and suddenly applied at $t=0$

$$
e_{s}=1(t) \begin{cases}=0 & t<0 \\ =1 & t>0\end{cases}
$$

We have the operational rule

$$
\begin{gathered}
\frac{p}{p+r} 1(t)=e^{-r t} \\
p 1(t)=\frac{d}{d t} 1(t)=\delta(t)
\end{gathered}
$$

Hence applying the complete operator $\bar{Q}$ as given by (17) to $e_{\text {a }}$ we find

$$
\sigma_{s}=\int_{0}^{\infty} e^{-r t} Q(r) \gamma(r) d r+Q+Q^{\prime} \delta(t)
$$

The time history of the stress contains a distribution of exponential stress relaxations. The Dirac function $\delta(t)$ corresponds to infinite shear rate at $t=0$. The coefficient $Q^{\prime}$ is equivalent to a viscosity coefficient since

$$
Q^{\prime} p e_{\mathrm{s}}=Q^{\prime} \frac{d e_{\mathrm{s}}}{d t}
$$

More generally, if the strain $e_{s}(t)$ is an arbitrary function of time the significance of the operator is given by

$$
\frac{p}{p+r} e_{s}(t)=e^{-r t} \int_{0}^{t} e^{r \tau} d e_{s}(\tau)
$$

The complete stress-strain law expressed by the operational equation (21) is therefore

$$
\frac{1}{2} \sigma_{s}=\int_{0}^{\infty} Q(r) \gamma(r) d r \int_{0}^{t} e^{-r(t-\tau)} d e_{s}(\tau)+Q e_{s}(t)+Q^{\prime} \frac{d e_{s}(t)}{d t}
$$

The coefficient $Q$ is pure elastic modulus. Putting

$$
\begin{gathered}
Q(r) \gamma(r)=F(r) \\
h(t)=\int_{0}^{\infty} F(r) e^{-r t} d r
\end{gathered}
$$

we write (28) in the form

$$
\frac{1}{2} \sigma_{s}=\int_{0}^{t} h(t-\tau) d e_{a}(\tau)+Q e_{s}(t)+Q^{\prime} \frac{d e_{s}(t)}{d t}
$$

which brings out an heredity function $h(t)$.

By the correspondence rule we may apply immediately the elastic-plate relations (14) to the case of a viscoelastic plate. The Lamé constants $G$ and $\lambda$ are replaced by the corresponding operators $\bar{Q}$ and $\bar{R}$. The coefficient $B$ becomes the operator.

$$
\bar{B}=\frac{4 \bar{Q}(\bar{Q}+\bar{R})}{2 \bar{Q}+\bar{R}}
$$

and equations (14) are transformed into the operational relations

$$
\begin{aligned}
q_{0} & =\left(\frac{1}{12} \bar{B} h^{2} l^{2}-P+\bar{Q}\right) h l^{2} V-2 \bar{Q} l U \\
2 \tau_{0} & =-2 \bar{Q} l V+\frac{4 \bar{Q}}{h} U
\end{aligned}
$$

Again, these may be considered as relations between Fourier or Laplace transforms or as differential and integral operations relating the quantities $U, V$, to $q_{0}$ and $\tau_{0}$.

We should finally add a remark concerning the deformation produced by the compressive load $P$. If the material tends to flow indefinitely under load the compression $P$ will be associated with a constant rate of compressional deformation of the plate. This deformation, however, will be disregarded as we are interested in cases where the deflection normal to the plate is the preponderant effect. 


\section{Deflection of a Semi-Infinite Viscoelastic Medium Under Normal and Tangential Surface Load}

We have pointed out that from the correspondence rule we may proceed exactly as in the theory of elasticity provided we replace the elastic coefficients by the corresponding operators. We shall therefore proceed directly by using operational equations throughout. The semi-infinite medium is represented in Fig. 2. It has a boundary at $y=0$ on which the normal and tangential loads are $q^{\prime}$ and $\tau$. The solid half space is in the region $y>0$. We write Lamés equations for the displacement components $u$ and $v$ of the solid after replacing the elastic coefficients $G$ and $\lambda$ by the operators $\bar{Q}$ and $\bar{R}$. We have

$$
\begin{aligned}
& \bar{Q} \nabla^{2} u+(\bar{Q}+\bar{R}) \frac{\partial e}{\partial x}=0 \\
& \bar{Q} \nabla^{2} v+(\bar{Q}+\bar{R}) \frac{\partial e}{\partial y}=0
\end{aligned}
$$

with

$$
\begin{aligned}
\nabla^{2} & =\frac{\partial^{2}}{\partial x^{2}}+\frac{\partial^{2}}{\partial y^{2}} \\
e & =\frac{\partial u}{\partial x}+\frac{\partial v}{\partial y}
\end{aligned}
$$

The Papkovitch-Boussinesq solution of these equations is

$$
\vec{u}=-\operatorname{grad}\left(\psi_{0}+\vec{r}_{0} \vec{\psi}\right)+2 \frac{(2 \bar{Q}+\bar{R})}{\bar{Q}+\bar{R}} \vec{\psi}
$$

The field displacement vector is

$$
\vec{u}=(u, v)
$$

and the co-ordinate vector is

$$
\vec{r}=(x, y)
$$

The vector $\vec{\psi}$ and the scalar $\psi_{0}$ satisfy Laplace's equation

$$
\nabla^{2} \psi_{0}=\nabla^{2} \vec{\psi}=0
$$

We shall choose the vector $\vec{\psi}$ to be oriented along $y$ and put

$$
\vec{\psi}=\left(0, \psi_{y}\right)
$$

The component $\psi_{y}$ also satisfied Laplace's equation

$$
\nabla^{2} \psi_{\nu}=0
$$

We are seeking here a solution which will match that of the plate

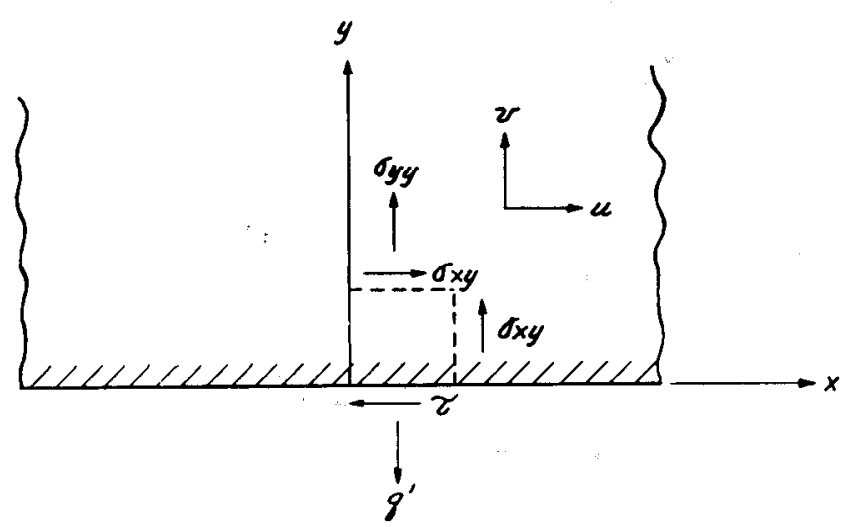

Fig. 2 Viscoelastic half-space at the boundary. We therefore assume a sinusoidal distribution of the surface load, i.e.

$$
\begin{aligned}
\tau & =\tau_{0} \sin l x \\
q^{\prime} & =q_{0}{ }^{\prime} \cos l x
\end{aligned}
$$

We also choose Boussinesq potentials $\psi_{0}$ and $\psi_{v}$ which contain the same trigonometric factors, and write

$$
\begin{aligned}
& \psi_{0}=A e^{-l y} \cos l x \\
& \psi_{y}=C e^{-l y} \cos l x
\end{aligned}
$$

These functions satisfy Laplace's equation in compliance with (38) and (40). Substituting $\psi_{0}$ and $\psi_{y}$ in (35) yields the displacement field,

$$
\begin{aligned}
u & =l(A+C y) e^{-l y} \sin l x \\
v & =\left\{l A+C\left[l y-1+\frac{2(2 \bar{Q}+\bar{R})}{\bar{Q}+\bar{R}}\right]\right\} e^{-l y} \cos l x
\end{aligned}
$$

From stress-strain relations (15) we derive the relevant stress components at the boundary $y=0$. We find

$$
\begin{gathered}
q^{\prime}=\sigma_{y y}=2\left[-A l-\frac{2 \bar{Q}+\bar{R}}{\bar{Q}+\bar{R}} C\right] Q l \cos l x \\
\tau=\sigma_{x y}=2\left[-A l-\frac{\bar{Q}}{\bar{Q}+\bar{R}} C\right] Q l \sin l x
\end{gathered}
$$

The displacement $u, v$ at the boundary is found by putting $y=0$ in (43). We write the boundary displacement as

$$
\begin{aligned}
& u=U \sin l x \\
& v=V \cos l x
\end{aligned}
$$

and the boundary stresses as

$$
\begin{aligned}
q^{\prime} & =q_{0}{ }^{\prime} \cos l x \\
\tau & =\tau_{0} \sin l x
\end{aligned}
$$

Then eliminating $A$ and $C$ in equations (43) and (44) (with $y=0$ ) yields the following relation between surface stress and displacement:

$$
\begin{aligned}
& \frac{1}{2 \bar{Q} l} q_{0}{ }^{\prime}=-\frac{\bar{Q}}{3 \bar{Q}+\bar{R}} U-\frac{2 \bar{Q}+\bar{R}}{3 \bar{Q}+\bar{R}} V \\
& \frac{1}{2 \bar{Q} l} \tau_{0}=-\frac{2 \bar{Q}+\bar{R}}{3 \bar{Q}+\bar{R}} U-\frac{\bar{Q}}{3 \bar{Q}+\bar{R}} V
\end{aligned}
$$

It is of interest to call attention to the particular case of the incompressible material. This is the case for which $\bar{R}=\infty$ while $e=0$ and $\bar{R} e$ remains finite. If we substitute $\bar{R}=\infty$ in relations (47) they simplify to

$$
\begin{aligned}
q_{0}^{\prime} & =-2 \bar{Q} l V \\
\tau_{0} & =-2 \bar{Q} l U
\end{aligned}
$$

In this case the vertical and horizontal displacements are uncoupled.

We must remember that the significance of these relations is operational. For instance, if the material is elastic and incompressible then relations (48) are proportionality relations with $\bar{Q}$ replaced by the shear modulus $G$. If the material is an incompressible viscous fluid of viscosity $\mu$ then

$$
\bar{Q}=\mu p .
$$

and equations (48) become differential relations 


$$
\begin{aligned}
q_{0}^{\prime} & =-2 \mu l \frac{d V}{d t} \\
\tau_{0} & =-2 \mu l \frac{d U}{d t}
\end{aligned}
$$

If the material is elastic in pure compression with an elastic hulk modulus $K$, we have shown [4] that the operator $\bar{R}$ is given in terms of $\bar{Q}$ by

$$
\bar{R}=K-\frac{2}{3} \bar{Q}
$$

In this case, even for a viscous fuid relations (47) imply both integrations and differentiations.

\section{Unstable Deformation of the Embedded Layer}

We are now in a position to consider the case of the embedded layer by putting together the foregoing results. We will first assume the case of perfect adherence between the layer and the embedding medium, Fig. 3. We will then follow with a brief discussion of the case of imperfect adherence.

Assuming perfect adherence the displacements $U$ and $V$ are equal at the interface for both media. The stresses also are continuous, the value of the shear $\tau_{0}$ being the same at each boundary and the normal load $q_{0}$ on the layer being equal to twice the normal traction $q_{0}^{\prime}$ at the boundary

$$
q_{0}=2 q_{0}^{\prime}
$$

From (32) and taking into account (51) we may write the stress at the houndary of the layer as

$$
\begin{aligned}
q_{0}{ }^{\prime} & =\left(\bar{A}_{11} V+\bar{A}_{12} U\right) l \\
\tau_{0} & =\left(\bar{A}_{12} V+\bar{A}_{22} U\right) l
\end{aligned}
$$

with the operators

$$
\begin{aligned}
& \bar{A}_{11}=1 / 2\left(1 / 12 \bar{B} h^{2} l^{2}-P+\bar{Q}\right) l h \\
& \bar{A}_{12}=-\bar{Q} \\
& \bar{A}_{22}=2 \bar{Q} / l h
\end{aligned}
$$

The stress at the boundary of the embedding medium is given by (47). We replace $\bar{Q}$ and $\bar{R}$ by $\bar{Q}_{1}$ and $\bar{R}_{1}$, the latter referring to the viscoelastic properties of the embedding medium while the former refer to the properties of the layer. We write, for the stress at the boundary of the half space,

$$
\begin{aligned}
q_{0}{ }^{\prime} & =-\left(\bar{C}_{1} V+\bar{C}_{2} U\right) l \\
\tau_{0} & =-\left(\bar{C}_{2} V+\bar{C}_{1} U\right) l
\end{aligned}
$$

with the operators

$$
\begin{aligned}
& \bar{C}_{1}=2 \bar{Q}_{1} \frac{2 \bar{Q}_{1}+\bar{R}_{1}}{3 \bar{Q}_{1}+\bar{R}_{1}} \\
& \bar{C}_{2}=2 \bar{Q}_{1} \frac{\bar{Q}_{1}}{3 \bar{Q}_{1}+\bar{R}_{1}}
\end{aligned}
$$

The condition that the stresses are continuous at the interface is obtained by equating expressions (52) and (54), i.e.

$$
\begin{aligned}
& \left(\bar{A}_{11}+\bar{C}_{1}\right) V+\left(\bar{A}_{12}+\bar{C}_{2}\right) U=0 \\
& \left(\bar{A}_{12}+\bar{C}_{2}\right) V+\left(\bar{A}_{22}+\bar{C}_{1}\right) U=0
\end{aligned}
$$

At this point we must call attention to a property of the operators which is implicit in some remarks made previously concerning their significance. All the operational equations derived here are valid if the variables vary with time proportionally to

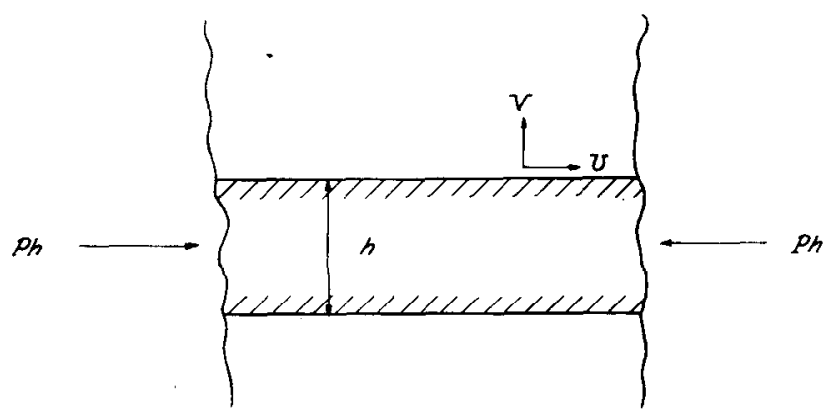

Fig. 3 Embedded layer

an exponential factor $e^{p t}$. In that case all operators are simply algebraic quantities and $p$ instead of being the differential operator $d / d t$ is the algebraic value of the coefficient of time in the exponential factor. If we now consider equations (56) we conclude that instability of the layer will appear whenever there are positive values of $p$ for which the determinant vanishes,

$$
\left|\begin{array}{ll}
\bar{A}_{11}+\bar{C}_{1} & \bar{A}_{12}+\bar{C}_{2} \\
\bar{A}_{12}+\bar{C}_{2} & \bar{A}_{22}+\bar{C}_{1}
\end{array}\right|=0
$$

In that case any initial value of $U$ and $V$ no matter how small will increase exponentially with time proportionally to $e^{p t}$. An instability of the layer will appear in the form of sinusoidal folding. In principle, a range of wave lengths will be unstable but not all wave lengths will grow at the same rate. For a given value of the load $P$ equation (57) may be considered as a relation between $p$ and $l h$. The latter parameter being related to the wave length $L$ of the folding by the relation

$$
\frac{L}{h}=2 \pi / l h
$$

The wave length for which the value of $p$ is maximum will tend to grow faster than others and will generally be the observed one. We have called it the "dominant wave length" $L_{d}$, the corresponding value of $l$ being denoted by $l_{d}$. We have discussed the behavior of the folding and the value of the dominant wave length for various types of malerials in reference [6] for the case of perfect slip. In the next section we shall discuss in more detail the case for which the layer and the embedding medium are both incompressible viscous solids with perfect adherence.

Before concluding this section let us formulate the case of imperfect adherence. In this case it is assumed that slipping may occur at the interface of the layer and the embedding medium. The slip layer is assumed to be a thin viscous layer of thickness $h$. and viscosity coefficient $\mu_{s}$. The interface displacements of the folding layer and the medium are denoted, respectively, by $U$ sin $l x$ and $U_{1} \sin l x$. The shearing stress $\tau_{0}$ at the interface is proportional to the rate of slip, according to the equation

$$
\tau_{0}=b p\left(U_{1}-U\right)
$$

with a coefficient of slip resistance

$$
b=\mu_{s} / h_{s}
$$

Expressing that there is continuity of stress and normal displacement $V$, we find the three equations

$$
\begin{gathered}
\left(\bar{A}_{11}+\bar{C}_{1}\right) V+\bar{A}_{12} U+\bar{C}_{2} U_{1}=0 \\
\left(\bar{A}_{12}+\bar{C}_{2}\right) V+\bar{A}_{22} U+\bar{C}_{1} U_{1}=0 \\
\bar{C}_{2} V-p \frac{b}{l} U+\left(\frac{p b}{l}+\bar{C}_{1}\right) U_{1}=0
\end{gathered}
$$


Equating to zero the determinant yields

$$
\begin{aligned}
& \bar{C}_{1}\left(\bar{A}_{11} \bar{A}_{22}-\bar{A}_{12}{ }^{2}\right)+\bar{A}_{22}\left(\bar{C}_{1}{ }^{2}-\bar{C}_{2}{ }^{2}\right) \\
& +\frac{p b}{l}\left[\left(\bar{A}_{11}+\bar{C}_{1}\right)\left(\bar{A}_{22}+\bar{C}_{1}\right)-\left(\bar{A}_{12}+\bar{C}_{2}\right)^{2}\right]=0
\end{aligned}
$$

The particular case of perfect adherence at the interface corresponds to $b=\infty$ giving again equation (57), while the case of perfect slip $b=0$ yields

$$
\bar{A}_{11}-\frac{\bar{A}_{12}{ }^{2}}{\bar{A}_{22}}+\bar{C}_{1}-\frac{\bar{C}_{2}^{2}}{\bar{C}_{1}}=0
$$

This case was considered previously [6]. We note that

$$
\bar{C}_{1}-\frac{\bar{C}_{2}^{2}}{\bar{C}_{1}}=\frac{2 \bar{Q}_{1}\left(\bar{Q}_{1}+\bar{R}_{1}\right)}{2 \bar{Q}_{1}+\bar{R}_{1}}=\frac{1}{2} \bar{B}_{1}
$$

while

$$
\bar{A}_{11}-\frac{\bar{A}_{12}^{2}}{\bar{A}_{22}}=\frac{1}{2}\left(\frac{1}{12} \bar{B} h^{2} l^{2}-P\right) l h
$$

Hence equation (63) becomes

$$
P=\frac{1}{12} \bar{B} h^{2} l^{2}+\frac{\bar{B}_{1}}{l h}
$$

It is readily seen that for every value of $p$ the load $P$ plotted as a function of $l h$ goes through a minimum which corresponds to the dominant wave length for that load. This last equation coincides with relation (3.1) of reference [6] where it was the object of a detailed discussion.

\section{The Case of Two Incompressible Viscous Solids}

We shall now consider the particular case of a layer of incompressible purely viscous solid embedded in a medium which is also incompressible and purely viscous. Perfect adherence is assumed at the interface. Incompressibility of the medium is expressed by the condition $\bar{R}_{1}=\infty$ hence from (55)

$$
\begin{aligned}
& \bar{C}_{1}=2 \bar{Q}_{1} \\
& \bar{C}_{2}=0
\end{aligned}
$$

Also for the layer

$$
\bar{B}=4 \bar{Q}
$$

For the case of pure viscosity the operators become

$$
\begin{aligned}
\bar{Q} & =\mu p \\
\bar{Q}_{1} & =\mu_{1} p
\end{aligned}
$$

where $\mu$ is the viscosity coefficient of the layer and $\mu_{1}$ that of the medium. The stability equation (57) becomes

$$
\left(\bar{A}_{11}+\bar{C}_{1}\right)\left(A_{22}+\bar{C}_{1}\right)-\bar{A}_{12}^{2}=0
$$

This yields

$$
P=\frac{1}{12} \bar{B} h^{2} l^{2}+\frac{4 \bar{Q}_{1}}{l h}+\frac{\bar{Q}_{1} l h}{1+\frac{\mu_{1}}{\mu} l h}
$$

The two first terms in this expression correspond to the case of perfect interfacial slip. The third term represents the correction due to perfect interfacial adherence.

Let us substitute expressions (68) and (69) for the operators. Equation (71) becomes

$$
\frac{P}{\mu p}=\frac{1}{3}(l h)^{2}+4 \frac{\mu_{1}}{\mu} \frac{1}{l h}+\frac{\mu_{1}}{\mu} \frac{l h}{1+\frac{\mu_{1}}{\mu} l h}
$$

The dominant wave length is determined by considering the minimum value of this expression as a function of $l h$. Equating to zero the derivative of this expression with respect to $l h$ yields an equation for the value $l_{d} h$ corresponding to the dominant wave length. We may write this equation

$$
\left(l_{d} h\right)^{3}=6 \frac{\mu_{1}}{\mu}\left[1-\frac{1}{4}\left(\frac{l_{d} h}{1+\frac{\mu_{1}}{\mu} l_{d} h}\right)^{2}\right]
$$

We note that the dominant wave length for the case of two incompressible viscous solids is independent of the load $P$. This is a particular case of a general rule for systems with a homogeneous spectrum as already discussed in reference (6).

The second term in the bracket of equation (73) represents the effect of the imperfect adherence at the interface. In order to evaluate the importance of this adherence let us consider the case of perfect interfacial slip. This corresponds to neglecting the second term in the bracket of equation (73). The equation then gives immediately the value of $l_{d} h$. Denoting by $l_{d}^{\prime}$ the value of $l_{d}$ for perfect slip we find

$$
l_{d}^{\prime} h=\left(\frac{6 \mu_{1}}{\mu}\right)^{1 / s}
$$

The corresponding dominant wave length for perfect slip is

$$
L_{d}^{\prime}=\frac{2 \pi}{l_{d}^{\prime}}=2 \pi h\left(\frac{\mu}{6 \mu_{1}}\right)^{1 / 2}
$$

We denote by

$$
L_{d}=\frac{2 \pi}{l_{d}}
$$

the dominant wave length for the case of perfect adherence as determined by equation (73). We may solve equation (73) for $l_{d} h$ as a function of $\mu / \mu_{1}$ and derive the value of $L / L_{d}{ }^{\prime}$. Values of this ratio are shown in Table 1 along with $l_{d}{ }^{\prime} h$ and $L^{\prime}{ }_{d} / h$.

Table 1

$\begin{array}{rcccr}\frac{\mu}{\mu_{1}} & l_{d}{ }^{\prime} h & L^{\prime}{ }_{d} / h & L_{d} / L_{d}{ }^{\prime} & \exp \left(p t_{1}\right) \\ 6 & 1.000 & 6.28 & 1.062 & 2.7 \\ 12 & 0.793 & 7.90 & 1.046 & 4.9 \\ 36 & 0.551 & 11.4 & 1.025 & 27.0 \\ 72 & 0.437 & 14.4 & 1.015 & 221 \\ 144 & 0.347 & 18.2 & 1.010 & 4440\end{array}$

Values of $L_{d} / L_{d}{ }^{\prime}$ show the influence of interfacial adherence on the dominant wave length. We can see that the effect of adherence is to lengthen the dominant wave length by amounts up to about 6 per cent. The effect however becomes vanishingly small for viscosity ratios $\mu / \mu_{\mathrm{I}}>70$. This corresponds to ratios of the dominant wave length to the thickness larger than 14.

That the wave length increases due to adherence follows immediately from inspection of equation (73) since the bracketed factor which represents the effect of adherence amounts to an increase in the viscosity $\mu$ of the layer.

As already pointed out in Section 1 , we have assumed that a shearing deformation occurs due to the interfacial stress $\tau$. If we neglect this shearing deformation, i.e., if we assume that all normal cross sections of the layer remain normal to the middle surface (neutral axis of the beam theory) this amounts to putting $\bar{Q}=\infty$ while retaining $\bar{B}$ finite in equation (70), hence replacing the bracket of relation (73) by

$$
\left[1-\frac{1}{4}\left(l_{d} h\right)^{2}\right]
$$


The correction of the dominant wave length due to adherence will in that case be increased, but only by an insignificant amount.

An important feature to be considered is the "degree of instability" as represented by the amplitude of the folding observed after a given time. Consider that a total time $t_{1}$ has elapsed such that under the load $P$ the compressive strain of the layer is $\epsilon$. We may write

$$
P=\frac{\bar{B}}{p} \frac{\epsilon}{t_{1}}=4 \mu \frac{\epsilon}{t_{1}}
$$

If the compressive strain is 25 per cent during this time $t_{1}$,

$$
P=\frac{\mu}{t_{1}}
$$

Let us go back to equation (72) and neglect the effect of the adherence, i.e., let us drop the third term in this cquation. Substituting in this equation the value (78) for $P$ we find

$$
\frac{1}{p t_{1}}=\frac{4}{12} \mu l^{2} h^{2}+\frac{4 \mu_{1}}{l h}
$$

Introducing the value $l=l_{d}^{\prime}$ from (74) corresponding to the dominant wave length yields

$$
p t_{1}=\frac{1}{\left(l_{d}^{\prime} h\right)^{2}}=\left(\frac{\mu}{6 \mu_{1}}\right)^{2 / 2}
$$

The factor $e^{p t_{1}}$ is an amplification factor. During a compressive strain of 25 per cent the amplitude of a dominant wave length initially present as an irregularity in the layer will be multiplied by $e^{p t_{1}}$. It may therefore be considered as a measure of the instability. Values of $e^{p t_{1}}$ are also shown in Table 1 against $\mu / \mu_{1}$.

We notice that the amplification factor $e^{p t_{1}}$ and hence the instability itself become large for values of the viscosity ratio $\mu / \mu_{1}$ of the order of 70 . For $\mu / \mu_{1}=70$ the dominant wave length is about 14 times the layer thickness. An initial disturbance of the layer with this wave length is amplified by a factor of about 200 in a compressive deformation of 25 per cent.

Another interesting point brought out by Table 1 is the fact that outside the range of the viscosity ratio $\mu / \mu_{1}$, for which the instability is mild, the influence of the interfacial adherence on the dominant wave length becomes negligible.

\section{Discussion of the More General Case}

In the previous section we have considered the folding of a viscous layer in a viscous medium. We shall now consider the case where one or both materials is not only viscous but viscoelastic in the more general sense. In order to simplify the derivation we shall neglect the influcnce of interface adherence. From our discussion in the previous section we may infer that neglecting adherence will not affect substantially the results. Since these cases have been treated in more detail in another publication [6], we shall simply outline briefly the results.

The essential result is furnished by equation (66) which gives $P$ as a function of $l h$ for every value of the rate coefficient $p$ in the time factor $\exp (p t)$. The variable $p$ appears in $\bar{B}$ and $\bar{B}_{1}$.

The dominant wave length $L_{d}$ is found by taking the derivative of equation (66) with respect to $l h$. We find

$$
L_{d}=2 \pi h\left(\frac{\bar{B}}{6 \bar{B}_{1}}\right)^{1 / 2}
$$

The corresponding load

$$
P=\frac{3}{2} \bar{B}_{1}\left(\frac{\bar{B}}{6 \bar{B}_{1}}\right)^{1 / 3}
$$

Eliminating $p$ between those two rclations yields the dominant wave length as a function of the load $P$. It can be seen that, in general, there is a lower eritical load obtained by putting $p=0$ in equation (82). At this load instability is incipient. The higher critical load is a buckling load obtained by putting $p=\infty$. This load corresponds to elastic buckling. The case of two incompressible viscous fluids examined in the foregoing corresponds to

$$
\begin{aligned}
\bar{B} & =4 \mu p \\
\bar{B}_{1} & =4 \mu_{1} p
\end{aligned}
$$

We find expression (75) independent of $p$. Hence in this case the dominant wave length is independent of the load.

For an elastic layer in a viscous medium both incompressible we have

$$
\begin{gathered}
\bar{B}=4 G \\
\bar{B}_{1}=4 \mu_{1} p
\end{gathered}
$$

where $G$ is the shear modulus of the layer. Applying expressions (81) and (82) and eliminating $p$ between them we find

$$
L_{d}=\pi h\left(\frac{4 G}{P}\right)^{1 / 2}
$$

In this case the dominant wave length decreases as the load increases. It is interesting to compare $L_{d}$ with the wave length of elastic buckling of the layer assumed free. It is found that $L_{d}$ is $\sqrt{ } 3$ times the wave length of free elastic buckling under the same load $P$. It is independent of the viscosity.

Another case is that of a viscous layer in an elastic medium. The layer of course must be sufficiently viscous to sustain the load. Assuming again incompressibility of both media, we find

$$
r_{d}=\frac{4}{3} \pi h \frac{P}{4 G_{1}}
$$

where $G_{1}$ is the shear modulus of the surrounding medium. The dominant wave length $(86)$ is independent of the viscosity and increases with the load.

The case of two Maxwell media is also examined in reference [6] and it is found that the ratio of relaxation times of the two media is an important parameter which controls the dependence of the dominant wave length on the load.

\section{Nonlinear and Incremental Stress-Strain Relations}

As mentioned in the previous work [6] there is a lot more to be said if we wish to apply irreversible thermodynamics to relations between incremental stresses and incremental strains. This is of course closely related to the problem of nonlinear rheology since a deformation may be considered as a succession of incremental states. While this appears to be quite a complex problem in general there is, however, one case which can be immediately analyzed by combining the thermodynamics of linear rheology [4] with those of the theory of elasticity for incremental stresses $[8,9]$. Consider a solid which is in a state of thermodynamic equilibrium under a stress field $S_{i j}$. Then for small deviations from this equilibrium state we may assume Onsager's relations to apply to the incremental strains and stresses. We must be careful, however, to express the stress components $t_{i j}$ as forces per unit area before incremental deformation $[8,9]$. This is necessary because application of thermodynamic principles requires the use of conjugate variables in such a way that

$$
W=\frac{1}{2} e_{i j} t_{i j}
$$

- The somewhat arbitrary assumption proposed by Ziegler [11] that linear viscoelasticity is directly applicable to plasticity overlooks the asymmetry of the operators. 
represents the work done by $t_{i j}$ with $e_{i j}$ the incremental strains. Then the relation between $e_{i j}$ and $t_{i j}$ is of the same general type as for the unstressed system

$$
t_{i j}=P_{i j}{ }^{k l} e_{k l}
$$

where $P_{i j}{ }^{k l}$ is the tensor operator derived in reference [4].

The actual incremental stresses $\sigma_{i j}$ as referred to areas after deformation are related to $t_{i j}$ by the relations [9]

$$
t_{i j}=\sigma_{i j}+e S_{i j}-\frac{1}{2}\left(e_{i l} S_{j l}+e_{j l} S_{i l}\right)
$$

which may also be written

$$
t_{i j}=\sigma_{i j}+B_{i j}{ }^{l k} e_{l k}
$$

with

$$
\begin{aligned}
B_{i j}{ }^{l k}=\delta_{l k} S_{i j} & -\frac{1}{2}\left(\delta_{i k} S_{j l}+\delta_{j k} S_{i l}\right) \\
& -\frac{1}{2}\left(\delta_{i l} S_{j k}+\delta_{j l} S_{i k}\right)
\end{aligned}
$$

Note that this is not generally a symmetric tensor, i.e.,

$$
B_{i j}{ }^{l k} \neq B_{l k}^{i j}
$$

For instance, if we start from a two-dimensional state of principal stresses

$$
S_{i j}=\left\|\begin{array}{ll}
S_{12} & 0 \\
0 & S_{22}
\end{array}\right\|
$$

Then

$$
\begin{aligned}
& B_{11}{ }^{22}=S_{22} \\
& B_{22}{ }^{21}=S_{11}
\end{aligned}
$$

Hence the tensor is symmetric only if we have

$$
S_{22}=S_{11}
$$

i.e., if the initial state is isotropic. In three dimensions this will be the case if the initial stress is hydrostatic. Substitution of (90) in (88) yields

$$
\sigma_{i j}=\left(P_{i j}^{l k}+B_{i j}{ }^{l k}\right) e_{l k}
$$

We conclude that under our assumptions the relation between the actual incremental stress and strain is not, in general, expressed by a symmetric tensor operator except if the initial stress is hydrostatic.

We see therefore that the stress-strain relation (15) which involves a symmetric matrix is in contradiction with (96). The error, however, will be small if the initial stress is sufficiently small relative to the incremental matrix $P_{i j}{ }^{l k}$ (incremental moduli in the elastic case). This contradiction does not arise if the material is not assumed to satisfy the condition that it is initially in thermodynamic equilibrium subject to small perturbations, e.g., if the state of prestress produces a steady state of flow.
Similar considerations may be applied to the more general case of perturbation in the vicinity of a steady or near-steady creep. In this case, the creep rate is coupled to the deformation because of temperature changes due to the thermoelastic effect ${ }^{5}$ and the Onsager relations are not satisfied for the incremental quantities.

If the body is such that for infinitely slow deformations it becomes elastic whether linear or nonlinear, then it is probably legitimate to assume that Onsager's relations are satisfied for incremental deformations and to use relations of the type (96). The operator, however, is restricted in this case and must be such that an incremental stress produces no steady-state creep. Hence it must be of a form such that

$$
P_{i j}{ }^{l k} \neq 0 \text { for } p=0
$$

These simple remarks are presented here to indicate some of the deeper implications which are involved if we wish to extend linear viscoelasticity to incremental deformations.

\section{References}

1 P. P. Bijlaard, "On the Elastic Stability of Thin Plates Supported by a Continuous Medium," Proc. Koninklijke Nederlandsche Akademie van Wetenschappen, vol. 49, 1946, pp. 1189-1199.

2 P. P. Bijluard, "On the Elastic Stability of Sundwich Plates," Proc. Koninklijke Nederlandsche Akademie van Wetenschappen, vol. 50, 1947, pp. 79-193.

3 G. S. Gough, C. F. Elam, and N. A. De Bruyne, "The Stabilization of a Thin Sheet by a Continuous Supporting Medium," Journal of The Royal Aeronautical Society, vol. 44, 1940, pp. 12-43.

4 M. A. Biot, "Theory of Stress-Strain Relations in Anisotropic Viscoelasticity and Relaxation Phenomena," Journal of Applied Physics, vol. 25, 1954, pp, 1385-1391.

5 M. A. Biot, "Variational and Lagrangian Methods in Viscoelasticity," Proceedings IUTAM Colloquium on the Deformation and Flow of Solids, Madrid, 1955, Springer, Berlin, 1956.

6 M. A. Biot, "Folding Instability of a Layered Viscoelastic Medium under Compression," Proceedings of the Royal Society, series A, vol. 242, 1957, pp. 444-454.

7 M. A. Biot, "Folding of a Layered Viscoelastic Medium Derived From an Exact Stability Theory of a Continuum Under Initial Stress," Quarterly of Applied Mathematics, vol. 17, 1959, pp. 185-204.

8 M. A. Biot, "Nonlinear Theory of Elasticity and the Linearized Case for a Body Under Initial Stress," Philosophical Magazine vol, 27,1939 , pp. 468-489.

9 M. A. Biot, "Elastizitats Theorie Zweiter Ordnung mit Anwendungen," Zeitschrift für angewandte Mathematik und Mechanik, vol. 20, 1940, pp. 89-99.

10 R. Mazet, "On a Suitable Model for Thermoelastic Vibrations of a Test Specimen Undergoing Creep," Ninth International Congress of Applied Mechanics, Brussels, 1956.

11 H. Ziegler, "An Attempt to Generalize Onsager's Principle and its Significance for Rheological Problems," Technical Report No. 13, Division of Applied Mathematics, Brown University, March 1957.

12 J. N. Goodier, "Cylindrical Buckling of Sandwich Plates," Journal of Applied Mechanics, vol. 13, Trans. ASME, vol. 68, 1946, pp. 253-260

13. M. A. Biot, "The Influence of Gravity on the Folding of a Layered Viscoelastic Medium Under Compression," Journal of the Franklin Institute, vol. 267, 1959, pp. 211-228.

${ }^{5}$ Equations for one-dimensional oscillations of a simple model of this type were given by Mazet [10]. 